\title{
Capturing, Restoring, and Presenting, the Independent Radar Investigation System (IRIS)
}

\author{
Benjamin Trethowan \\ The National Museum of Computing, Block H, Bletchley Park, Milton Keynes, \\ Buckinghamshire, United Kingdom \\ ben.trethowan@tnmoc.org
}

\begin{abstract}
This paper describes a recent project at The National Museum of Computing (TNMoC) to capture, restore to working order, and present to the public, an early air traffic control system. It discusses the importance of capturing an extensive range of information, relating to the system, at the point of donation, the value of this information within the restoration process, the techniques used within the restoration itself, and the value of expressing the social impact of the system in order to convey its relevance to the public.
\end{abstract}

Keywords: ANSP, ASTERIX, ATS, BEG, CAA, CCC, DEC, DEG, IRIS, LATCC, NATS, NERC, PDP, PRDS, RARE, RBEG, RDI, RDP, TNMoC.

\section{Introduction}

The Independent Radar Investigation System (IRIS) is an isolated Radar Data Processing (RDP) system, originally used for the investigation of air traffic incidents. It allowed recorded radar and voice (i.e. radiotelephony) data to be replayed together, in synchronisation, for investigative purposes. It was designed and built in the early 1970s at the London Area and Terminal Control Centre (LATCC) in West Drayton, Middlesex, on behalf of National Air Traffic Services (NATS); the UK's Air Navigation Service Provider (ANSP) for all en-route, and a large proportion of terminal, Air Traffic Services (ATS).

The IRIS was in use until the LATCC facility was finally decommissioned in January 2008, when air traffic operations transferred to the New En-Route Centre (NERC) facility in Swanwick, Hampshire. During its operational lifetime, the LATCC facility handled over 48 million aircraft movements.

Prior to the decommissioning of the LATCC facility, NATS had approached The National Museum of Computing (TNMoC) in order to donate the IRIS. It was felt that the IRIS represented a key element of the history of the UK's transport infrastructure, as well as an excellent example of early British computer engineering. TNMoC accepted the IRIS into its collection in April 2008, following its disassembly; packaging, and transport from the LATCC site in March 2008. Both organisations were keen that TNMoC should restore the IRIS to full working order, such that the system could be demonstrated for the enjoyment of the visiting public. 
Between May and September 2008, a small team of volunteers at TNMoC carefully reassembled and restored the IRIS. Restoration activity was completed in October 2008, when the IRIS completed its first successful playback after departing the LATCC facility.

\section{Background}

The IRIS system is itself a small, standalone installation of a much larger RDP system, the Processed Radar Display Subsystem (PRDS). The operational requirement of PRDS was to "accept radar plot and label data, flight plan data, etc. and to process and display this data."[1] In short, the PRDS provided air traffic controllers with their operational situation display, indicating the current position of all aircraft within the selected radar range and system coverage.

The PRDS was not the primary means of radar data processing, but provided a bypass function "intended to provide an alternative radar display service when the Central Computer Complex (CCC) is unavailable."[1] In short, the PRDS accepted processed radar data, from a primary source, and displayed this to an air traffic controller during normal operations. However it also provided an alternative means of processing radar data, known as 'bypass', should the primary source fail for whatever reason.

The IRIS is composed of a small subset of the PRDS subsystems, representing the smallest / simplest collection of subsystems necessary to generate an operational situation display from recorded radar data. The IRIS is capable of processing a maximum of three radar data channels simultaneously, and displaying a maximum of two of these channels, on an operational situation display, at any one time.

The PRDS system architecture, and hence the IRIS system architecture, is based upon the use of the Digital Equipment Corporation (DEC) Programmed Data Processor (PDP) - 11 range. The IRIS uses four such PDP-11 processors, as follows; two PDP-11/34 processors for the Display Equipment Groups (DEGs), used to render vector graphics onto two operational situation displays, one PDP-11/84 processor for the Bypass Equipment Group (BEG), used to process two radar data channels, and one PDP-11/84 processor for the Redundant Bypass Equipment Group (RBEG), used to process one radar data channel and control / monitor all other systems.

To achieve playback within the IRIS; recorded radar data is fed into the RBEG and BEG systems for processing, the processed radar data is then passed onto the DEG systems for rendering, with the vector graphics commands then being passed onto the operational situation display (also known as the Sector Equipment Group (SEG)) for drawing onto the screen.

Both the RBEG and BEG systems continually process incoming radar data, regardless of the channel; range, or other selection made at the operational situation display. The DEG systems react to input from the operational situation displays, and are responsible for rendering the display in accordance with the selected channel; dual-channel, range, map overlay, and other user-selectable settings. 


\section{Capture}

At the time of the original donation, both NATS and TNMoC were keen to ensure that as much supporting material / additional artefacts, relating to the IRIS, were captured as soon as possible. The LATCC site was due to be demolished shortly after the facility was vacated, so the only opportunity to accept and preserve this supporting material would have to be taken at the earliest possible stage. The exploitation of this opportunity, and the broadness of the material captured, later proved to be a vital element in achieving the successful restoration of the IRIS.

One of the first issues experienced during the restoration, which was only resolved as a result of the original broad capture, was that of bespoke system design. Despite the fact that the IRIS subsystems utilised a popular computing platform of the period (DEC PDP-11), it transpired that a large amount of the hardware within these platforms was either of a bespoke design, or had been modified. One such example concerned the Radar Data Interface (RDI) hardware contained within the RBEG and BEG systems. Whilst the RDI hardware was modeled on a familiar full-size UNIBUS card, for obvious reasons, the microelectronics upon the card were developed specifically for the PRDS by the systems' original supplier, Plessey. Initial problems with the RDI hardware, relating to component failure, were only resolved during the restoration process due to the availability of the original Plessey schematics, which had been captured alongside the IRIS at the point of donation. Had this information not been captured when it was, it would have long since been lost; given that the demolition of the LATCC site would have progressed several months by this stage. Considering also that the PRDS implementation was unique, there would have been no alternative source for this information.

A similar issue experienced during the restoration, resolved again by the availability of supporting material, was that of legacy proprietary data communication protocols. The UK Civil Aviation Authority (CAA) had developed a proprietary protocol for the exchange of radar data, based upon a synchronous serial method of communication. The IRIS subsystems were designed to accept radar data only in this format, and were not able to process more recent radar data formats such as the All Purpose Structured Eurocontrol Surveillance Information Exchange (ASTERIX) format. The need to monitor, test, and generate, radar data for the IRIS RDP subsystems, spawned a further need to first understand the CAA radar data format itself. Luckily, the original specification for this radar data format, issued by the CAA, had also been captured alongside IRIS at the point of donation.

In addition to the engineering issues mentioned previously, the successful restoration was also dependent upon gaining an acceptable appreciation of the complex operational context within which the IRIS system originally operated. The PRDS subsystems, from which IRIS is built, originally interfaced with a multitude of additional operational systems, performing functions such as aeronautical messaging; code / callsign conversion, meteorological data communication, and flight plan processing, etc. It was therefore necessary to understand how the IRIS subsystems should operate without the support of these additional systems, and how that might affect the behaviour that the IRIS would exhibit in its initial stages of operation, 
following restoration. Again, the initial capture had managed to preserve complete PRDS schematics, including training course material on how the PRDS operated with the additional LATCC systems. Without the availability of this sort of documentation, the successful restoration of the IRIS almost certainly would not have been possible.

\section{Restoration}

As well as reaping the benefits of a broad capture at the point of donation, the restoration team also utilised a number of other techniques to combat additional challenges and ensure success.

Unfortunately, it is almost never possible to capture absolutely everything that might be required to facilitate a successful restoration. Setting aside the common issues of storage capacity, transport costs, and underfunding, it is often the case that some supporting artefacts have already been lost; prior to the initial offer of donation being made.

For example, the technical documentation associated with a radar data recording and replay system, supplied alongside the IRIS, had already been lost; prior to the removal of the IRIS and its associated artefacts in April 2008. This recording and replay system, known as RARE, would later prove to be an essential element in achieving the successful restoration of the IRIS, by acting as a source of the CAA format radar data necessary to achieve playback. A number of issues were initially encountered during the restoration of the RARE system, many associated with the total lack of documentation and / or understanding of how the system should respond in certain situations, or when given certain stimuli. In an attempt to combat these issues, the restoration team contacted the original donor, NATS; made a number of external enquiries, and conducted a small amount of research, in order to trace a technical contact for the RARE system that might be able to provide the necessary information. After a number of weeks, an individual who had not only worked for the original manufacturer of the RARE system, but who had also been personally involved in its design, contacted TNMoC to offer his assistance. The information that this individual was able to provide, either in hard copy form, or through technical interchange meetings, led to the successful restoration of the RARE system, and subsequently the IRIS.

A similar situation was encountered in relation to the IRIS subsystems themselves. In this case, the issue was not that the supporting material had not been captured, or lost, but that it simply did not exist. A recurring issue, observed within the power supply units of some of the IRIS subsystems, did not appear to have been documented within any of the material available to the restoration team. After extensive investigations, the restoration team contacted the original donor, NATS, in order to ascertain whether any similar issues had been observed whilst the system was in service at the LATCC facility. Following discussions with NATS, it transpired that the issue we were observing was, in fact, a known one; and was the result of incorrect technical documentation. Whilst many of the existing NATS engineers were aware of this issue, and its cause, this information was not passed on to TNMoC at the point of 
donation as it had, quite simply, been forgotten about. The support of the original donor, and existing third-party suppliers, proved to be vital in these circumstances.

\section{$5 \quad$ Challenges}

Whilst many of the challenges encountered during the restoration process were resolved through the use of supporting material, or through contact with the original donor and its third-party suppliers, some could only be resolved through the successful application of engineering best practice.

One such challenge was that of degraded magnetic tape media, a common feature of many computer restoration projects. An additional radar data recording and replay system, known as the SE7000, had been supplied alongside the IRIS and accompanied by a number of radar data recordings on multi-track magnetic tape. The SE7000 is, quite simply, a multi-track magnetic tape deck, with multiple read / write heads that are used to record / replay multiple radar channels simultaneously. When inspecting the magnetic media that we had been given, it became apparent that the media was suffering from the common problem of oxide separation. The restoration team was fortunate enough to have been given some additional media however, which did not exhibit the separation issue. Thankfully, this has meant that we have been able to stop short of attempting to restore the degraded media, using a potentially hazardous process, for the time being.

Similar degradation issues were found to have affected the drive mechanism itself, with numerous rubber capstans and drive belts, etc. having perished. Contemporary replacement parts were found to resolve these issues however, allowing us to operate the SE7000 unit temporarily, in order to capture the radar data from any operable media onto a more suitable modern format.

Interestingly, the more recent RARE system suffered from similar issues, despite its use of closed tape cartridges (Exabyte) instead of open tape reels. A series of recurring issues were encountered with the Exabyte drive mechanisms, which had not been maintained since their withdrawal from service many years before the point of donation. A lack of suitable spares further exacerbated this problem, and led to the decision to immediately retire the RARE unit; following the successful capture of radar data from any operable media onto a more suitable modern format.

\section{Social Impact}

After defeating the many challenges of restoration, and successfully demonstrating the IRIS in full working order since October 2008, it is perhaps worth considering some of the motivations behind this project.

The IRIS offers us a unique opportunity to convey the impact that computing technology has had upon society, demonstrating why the history and development of computing is relevant to our visiting public. Our visitors have the opportunity to both study and interact with something that was a vital component of the UK's transport 
infrastructure for over 25 years, something that they can immediately identify with if they have ever flown away on holiday, or to a business meeting, etc.

It also delivers a significant visual impact as an operational exhibit; its size, and 'retro' appearance, provide a visually captivating display, far removed from the socalled 'beige boxes' that many of our visitors expect to see.

And finally, it presents us with an additional educational opportunity to captivate the many young people that visit our museum, and explain just how the development of computing technology has kept, and continues to keep, us safe in the sky.

\section{Reference}

1. National Air Traffic Services: Processed Radar Display Sub-system for the Civil Aviation Authority, vol. 1, RSL 1631, CRSS/A1/01 (April 1975) 